\title{
Successful Treatment of Refractory Squamous Cell Cancer of the Head and Neck with Nivolumab and Ipilimumab
}

\author{
Katjana S. Schwab ${ }^{a} \quad$ Glenn Kristiansen $^{b}$ Hans H. Schild ${ }^{c}$ \\ Stefanie E.A. Held ${ }^{a}$ Annkristin Heine ${ }^{a}$ Peter Brossart ${ }^{a}$ \\ ${ }^{a}$ Department of Internal Medicine 3, University Hospital Bonn, Bonn, Germany; \\ ${ }^{b}$ Institute of Pathology, University Hospital Bonn, Bonn, Germany; 'Department of \\ Radiology, University Hospital Bonn, Bonn, Germany
}

\section{Keywords}

Nivolumab · Ipilimumab · Squamous cell carcinoma $\cdot$ Anti-PD-1 inhibitor · Anti-CTLA4 antibody $\cdot$ Anti-programmed cell death protein 1 inhibitor

\begin{abstract}
Treatment options for patients with platinum-refractory, recurrent, metastatic head and neck squamous cell carcinoma (HNSCC) are limited, and prognosis is poor. Nivolumab (Opdivo) has been approved by the US Food and Drug Administration (FDA) for the treatment of patients with recurrent or metastatic HNSCC who have disease progression on or after platinum-based therapy. Recently, in patients with metastatic malignant melanoma a significant improvement of outcome and response was achieved with the combination of ipilimumab (CTLA4 antibody) and the programmed death (PD)-1 inhibitor nivolumab compared with monotherapy. Based on these results, the combination of nivolumab and ipilimumab has been approved by the FDA for the treatment of patients with unresectable or metastatic melanoma. So far, there have been no data concerning the combination of nivolumab and ipilimumab in squamous cell head and neck cancer. We here present the case of a 46-yearold male with refractory squamous cell head and neck cancer, who was successfully treated with the PD-1 inhibitor nivolumab in combination with the anti-CTLA4 antibody ipilimumab.




\section{Case Reports in Oncology}

\section{Introduction}

Squamous cell carcinoma of the head and neck accounts for $90 \%$ of all cases of carcinoma of the head and neck [1].

Treatment options for patients with platinum-refractory, recurrent, metastatic head and neck squamous cell carcinoma (HNSCC) are limited, and prognosis is poor. The recent CheckMate 141 clinical trial demonstrated that nivolumab, an anti-programmed cell death protein 1 monoclonal antibody, was efficacious in extending the median overall survival in this patient population compared with standard therapies [2]. Based on these data, nivolumab (Opdivo) has been approved by the US Food and Drug Administration (FDA) for the treatment of patients with recurrent or metastatic HNSCC who have disease progression on or after platinum-based therapy [3]. Recently, data of the checkmate 069 study have shown significant improvements in objective response and prolonged progression-free survival with the combination of nivolumab plus ipilimumab compared with ipilimumab alone in patients with metastatic malignant melanoma [4].

Based on these results, the combination of nivolumab and ipilimumab has been approved by the FDA for the treatment of patients with unresectable or metastatic melanoma. So far, there have been no data concerning the combination of nivolumab and ipilimumab in squamous cell head and neck cancer.

\section{Case Report}

We present the case of a 46-year-old male with refractory squamous cell head and neck cancer, who was successfully treated with the programmed death (PD)-1 inhibitor nivolumab in combination with the anti-CTLA4 antibody ipilimumab. In December 2016, a low differentiated squamous cancer of the tongue pT1,pN2b, L1,V0, G3 was diagnosed. There was no sign of human papilloma virus infection. After R0 resection and neck dissection, he underwent adjuvant radiochemotherapy with cisplatin $35 \mathrm{mg} / \mathrm{m}^{2}$ weekly.

In April 2016, a CT scan of the neck showed significant cervical lymph node enlargement. A biopsy confirmed a lymph node metastasis of a squamous cell carcinoma due to the previous cancer. There were no signs of further metastases. The tumor was surgically not resectable, so the intensification of systemic chemotherapy was performed with 5-FU, cisplatin, and cetuximab. A CT scan after two cycles revealed an unsatisfying response with stable disease (Fig. 1a).

The tumor was positive for PD ligand 1 (PD-L1) expression and due to the lack of other treatment options, a therapy with nivolumab $(3 \mathrm{mg} / \mathrm{kg}$ body weight every 2 weeks $)$ and ipilimumab (1 mg/kg every 6 weeks) was initiated in July 2016. Of note, the patient had a long history of juvenile idiopathic polyarthritis accompanied by unclassified autoimmune hepatitis. Ten days after start of therapy, an increase in rheumatoid factor and liver enzymes was detected. The MRI of the liver showed no pathologic findings, and the hepatitis serology was negative.

Because of a suspected underlying immuno-induced hepatitis, therapy with prednisolone was initiated (100 mg/day), and liver parameters decreased significantly. Nevertheless, administration of ipilimumab and nivolumab was continued, and 3 weeks after the second administration of ipilimumab, rheumatoid factor and liver enzymes increased but decreased again after restart of prednisolone $100 \mathrm{mg} /$ day. A CT scan 8 weeks after start of therapy 


\section{Case Reports in Oncology}

Schwab et al:: Successful Treatment of Refractory Squamous Cell Cancer of the Head and Neck with Nivolumab and Ipilimumab

showed a significant regression of tumor size, and 4 months after start of therapy in November 2016 (Fig. 1b), a nearly complete remission was achieved (Fig. 1c).

In January 2017, MRI showed signs of local relapse. Histology confirmed an infiltration of the known carcinoma. Thus, therapy with heavy ion radiation was initiated, and nivolumab monotherapy was continued. Follow-up with MRI in June 2017 showed stable disease.

Soluble PD-L1 and PD-L2 serum levels were evaluated before and during the treatment using a commercially available ELISA (R\&D Systems), showing a significant decrease in serum PD-L1 due to the response to the therapy, followed by an increase at the time of progression. There was no change in PD-L2 serum levels corresponding to response or progression to therapy.

Considering tumor biopsies, there was no change in PD-L1 or HLA class I expression during therapy. Of note, during therapy, no significant changes in white blood cells, B/T cells, T-helper cells, cytotoxic and regulatory T lymphocytes, NK cells, as well as myeloid-derived suppressor cells were detected.

\section{Discussion}

To our knowledge, this is the first case report showing a clinically meaningful response to nivolumab in combination with ipilimumab of a refractory metastatic squamous cancer of the head and neck. However, the above strategy, due to very high toxicity, has limitations for use in all patients [5, 6]. In our patient, despite the history of autoimmune hepatitis and juvenile polyarthritis, the treatment-induced elevation of liver enzymes was reversible under corticosteroids, and other side effects were moderate.

We observed that soluble PD-L1 serum levels decreased under response to therapy and increased at time of progression under treatment, while soluble PD-L2 serum levels stayed stable. Further studies are required to examine the value of soluble PD-L1 serum levels as a predictive marker of the response to immunotherapy.

In our patient treated with the combination of nivolumab and ipilimumab, a complete remission after 4 months of therapy was achieved with moderate and reversible side effects. So, the combination of nivolumab and ipilimumab could be a promising option in refractory metastatic squamous cell cancer of the head and neck [7]. There are several trials underway comparing the efficacy of immuno-oncology approaches with standard chemotherapy, and we are eagerly awaiting the results.

\section{Statement of Ethics}

The authors have no ethical conflicts to disclose.

\section{Disclosure Statement}

The authors have no conflicts of interest to declare. 


\section{Case Reports in Oncology}

\begin{tabular}{|c|c|}
\hline Case Rep Oncol 2018;11:1 & \\
\hline DOI: 10.1159/000485562 & $\begin{array}{l}\text { (c) } 2018 \text { The Author(s). Published by S. Karger AG, Basel } \\
\text { www.karger.com/cro }\end{array}$ \\
\hline
\end{tabular}

Schwab et al:: Successful Treatment of Refractory Squamous Cell Cancer of the Head and Neck with Nivolumab and Ipilimumab

\section{Funding Sources}

None.

\section{References}

1 National Comprehensive Cancer Network website. NCCN Guidelines ${ }^{\circledR}$. Head and Neck Cancers. v1.2017. https://www.nccn.org/professionals/physician_gls/PDF/head-and-neck.pdf (accessed April 4, 2017).

-2 Kiyota N, Hasegawa Y, Takahashi S, Yokota T, Yen CJ, Iwae S, Shimizu Y, Hong RL, Goto M, Kang JH, Sum Kenneth Li W, Ferris RL, Gillison M, Namba Y, Monga M, Lynch M, Tahara M: A randomized, open-label, Phase III clinical trial of nivolumab versus therapy of investigator's choice in recurrent squamous cell carcinoma of the head and neck: a subanalysis of Asian patients versus the global population in checkmate 141. Oral Oncol 2017;73:138-146.

3 Nivolumab for SCCHN. US Food and Drug Administration Approved Drugs website. http://www.fda.gov/Drugs/InformationOnDrugs/ApprovedDrugs/ucm528920.htm. Updated November 10, 2016 (accessed November 10, 2016)

-4 Hodi FS, Chesney J, Pavlick AC, Robert C, Grossmann KF, McDermott DF, Linette GP, Meyer N, Giguere JK, Agarwala SS, Shaheen M, Ernstoff MS, Minor DR, Salama AK, Taylor MH, Ott PA, Horak C, Gagnier P, Jiang J, Wolchok JD, Postow MA: Combined nivolumab and ipilimumab versus ipilimumab alone in patients with advanced melanoma: 2-year overall survival outcomes in a multicentre, randomised, controlled, phase 2 trial. Lancet Oncol 2016;17:1558-1568.

-5 Pollack MH, Betof A, Dearden H, Rapazzo K, Valentine I, Brohl AS, Ancell KK, Long GV, Menzies AM, Eroglu Z, Johnson DB, Shoushtari AN: Safety of resuming anti-PD-1 in patients with immune-related adverse events (irAEs) during combined anti-CTLA-4 and anti-PD1 in metastatic melanoma. Ann Oncol DOI 10.1093/annonc/mdx642.

-6 Bowyer S, Prithviraj P, Lorigan P, Larkin J, McArthur G, Atkinson V, Millward M, Khou M, Diem S, Ramanujam S, Kong B, Liniker E, Guminski A, Parente P, Andrews MC, Parakh S, Cebon J, Long GV, Carlino MS, Klein O: Efficacy and toxicity of treatment with the anti-CTLA-4 antibody ipilimumab in patients with metastatic melanoma after prior anti-PD-1 therapy. Br J Cancer 2016;114:1084-1089.

7 Swanson MS, Sinha UK: Rationale for combined blockade of PD-1 and CTLA-4 in advanced head and neck squamous cell cancer - review of current data. Oral Oncol 2015;51:12-15.
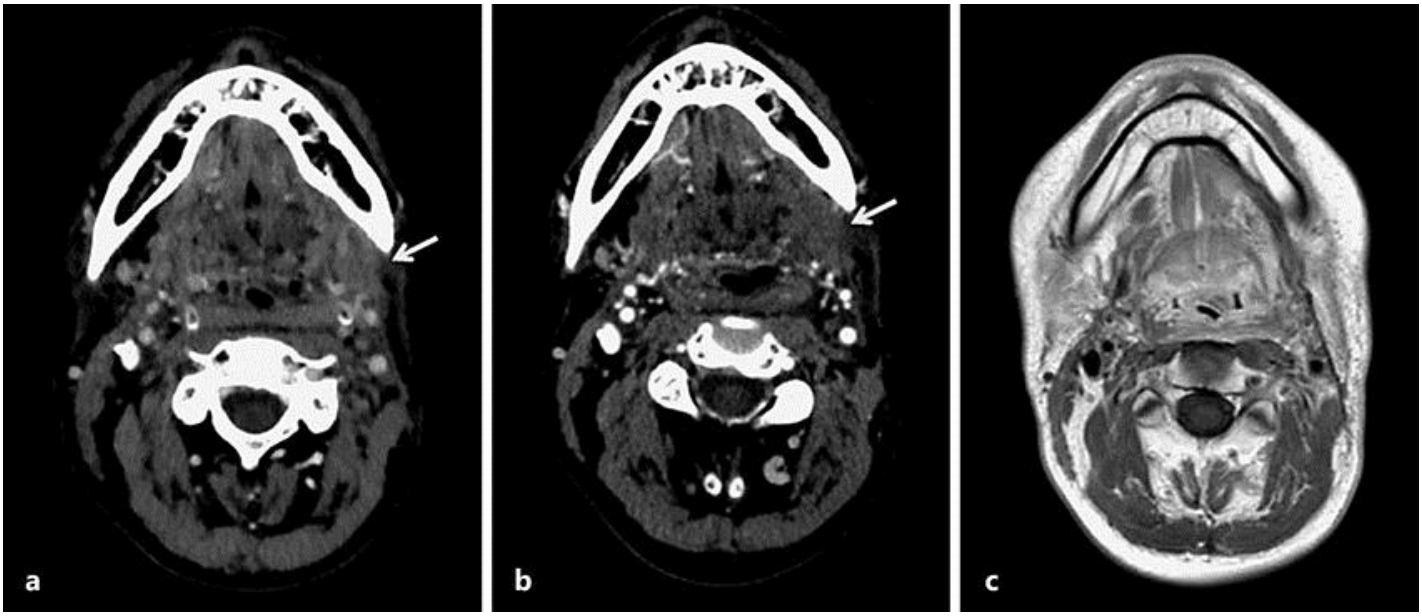

Fig. 1. CT before therapy (a), CT after 8 weeks of therapy (b), and MRI 4 months after start of therapy (c). Arrows show tumor manifestation. 